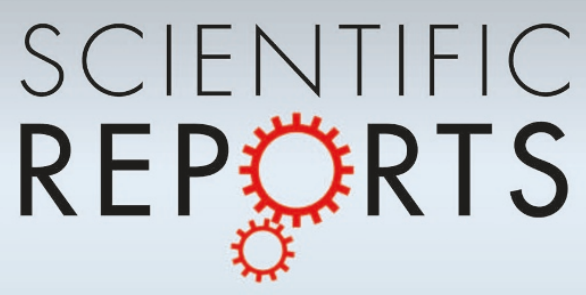

OPEN

SUBJECT AREAS:

ULTRAFAST LASERS

SEMICONDUCTOR LASERS

ULTRAFAST PHOTONICS

OPTICAL SPECTROSCOPY

Received

9 May 2014

Accepted

28 August 2014

Published

19 September 2014

Correspondence and requests for materials should be addressed to

S.Q.C. (sqchen@ee. ecnu.edu.cn)

* These authors contributed equally to this work.

\section{Direct generation of 2-ps blue pulses from gain-switched InGaN VCSEL assessed by up-conversion technique}

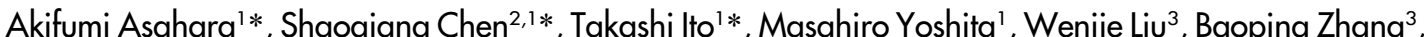
Tohru Suemoto' \& Hidefumi Akiyama'

\footnotetext{
${ }^{1}$ Institute for Solid State Physics, University of Tokyo, 5-1-5 Kashiwanoha, Kashiwa, Chiba 277-8581, Japan, ${ }^{2}$ Department of Electronic Engineering, East China Normal University, 500 Dongchuan Road, Shanghai 200241, China, ${ }^{3}$ Department of Physics \& Department of Electronic Engineering, Xiamen University, Xiamen 361005, Fujian, China.
}

Ultra-short pulses in blue region generated from compact and low-cost semiconductor lasers have attracted much attention for a wide variety of applications. Nitride-based vertical-cavity surface-emitting lasers (VCSELs), having intrinsic high material gain and short cavities, favor the generation of ultra-short blue pulses via a simple gain-switching technique. In this study, we fabricated a single-mode InGaN VCSEL consisting of 10-period InGaN/GaN quantum wells (QWs). The output pulses were evaluated accurately with an up-conversion measurement system having time resolution of $0.12 \mathrm{ps}$. We demonstrated that ultra-short blue pulses, as short as $2.2 \mathrm{ps}$ at $3.4 \mathrm{~K}$ and $4.0 \mathrm{ps}$ at room temperature, were generated from the gain-switched InGaN VCSEL via impulsive optical pumping, without any post-processing. The gain-switched pulses we obtained should greatly promote the development of ultra-short blue pulse generation. In addition, this successful assessment demonstrates the up-conversion technique's usefulness for characterizing ultra-short blue pulses from semiconductor lasers.

$\mathrm{N}$ itride-based blue semiconductor lasers have recently taken on an important role in optical science and technology ${ }^{1-5}$. In particular, picosecond optical pulses in the blue-wavelength band have demonstrated their potential for a wide range of applications $s^{6-10}$, including optical storage, bioimaging, and timeresolved spectroscopy. As a possible pulsed light source, gain-switched semiconductor lasers ${ }^{11-13}$ have attracted considerable attention for their advantages of low cost, simple operation, and compact size. To increase the range of such devices' applications, the gain-switched pulses need to be shortened to several picoseconds or less. Since the typical durations of gain-switched pulses lie within a range of several tens or hundreds of picoseconds, a number of post-processing techniques ${ }^{14-16}$ such as spectral filtering, pulse shaping, and pulse compression have been developed in order to shorten the pulse width. The simplest method, however, is the direct generation of ultra-short pulses from gain-switched semiconductor lasers without any post-processing.

The pulse width of gain-switched pulses is determined by the rise and decay times. Previous studies of ours ${ }^{17}$ have shown that the rise time is mainly limited by the saturation gain of the semiconductor laser, while the decay time is mainly limited by photon lifetime in the cavity. Therefore, semiconductor lasers with high optical gain and short cavity lifetime, such as vertical-cavity surface-emitting lasers (VCSELs) ${ }^{18-20}$, are favorable for the generation of short pulses via gain-switching. Additional advantages of VCSELs, such as single-longitudinal-mode operation, a low lasing threshold, high intrinsic bandwidth, a circular output beam with low divergence, wafer-scale processing, and the capability to form two-dimensional device arrays, have made VCSELs into very attractive devices.

On the other hand, widely used time-resolved measurement systems with temporal resolution longer than several picoseconds, such as streak cameras, high-speed photo-detectors, or oscilloscopes, are inadequate for the detailed assessment of pulses as short as picoseconds or less. Therefore, the development of accurate measurement systems with even finer time resolution is required. In existing time-resolved luminescence spectroscopy, the up-conversion (cross-correlation) method is a particularly promising candidate for achieving subpicosecondscale resolution. Besides its fine time resolution, this method has the advantage of a high signal/noise $(\mathrm{S} / \mathrm{N})$ ratio, because the signal is amplified by gate pulses during the up-conversion process. This technique has often been used in studying ultrafast phenomena in solids in fundamental physics research ${ }^{21,22}$ and in assessing the pulselasing properties of semiconductor lasers ${ }^{23,24}$. Although the up-conversion technique has not been applied to 
a
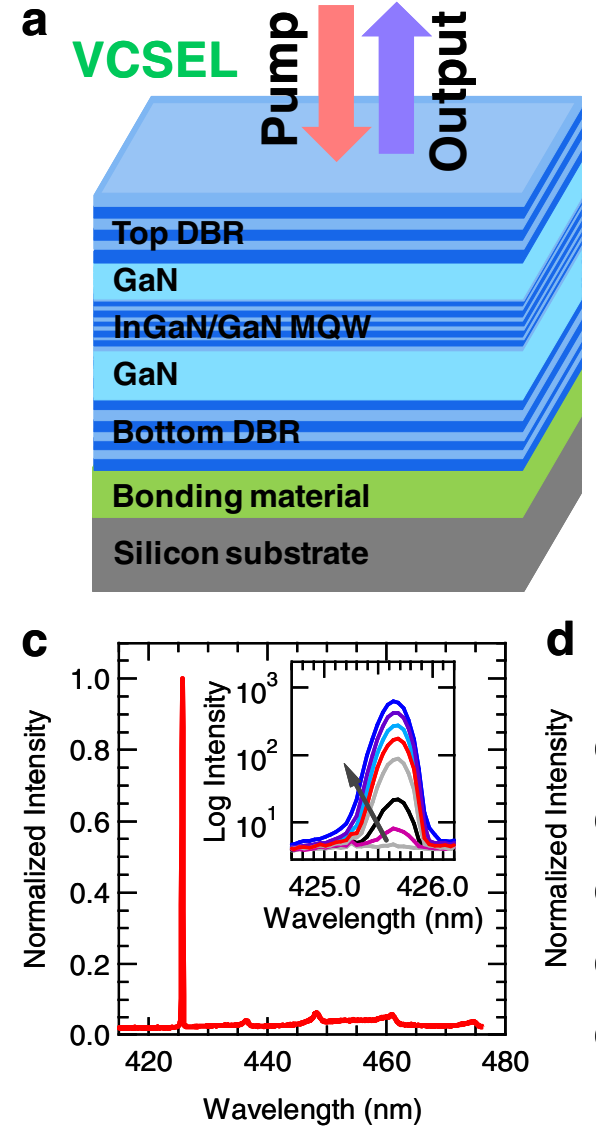
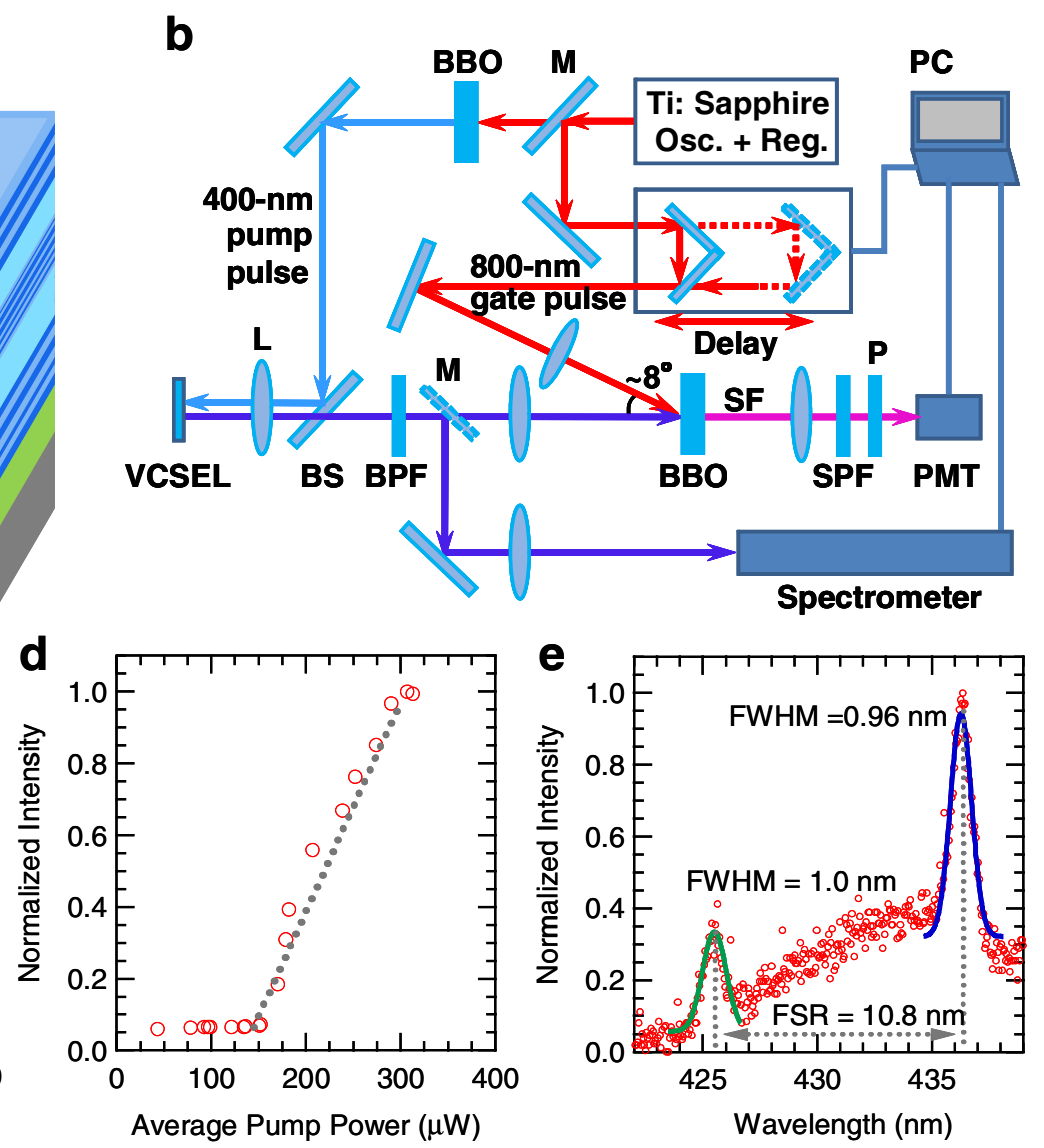

Figure $1 \mid$ (a) Schematic of the InGaN VCSEL structure. (b) Experimental apparatus. Osc.: Ti:sapphire oscillator; Reg.: regenerative amplifier; M: mirror; L: lens; BS: beam splitter; $\mathrm{BBO}: \beta-\mathrm{BaB}_{2} \mathrm{O}_{4}$ crystal; SF: sum-frequency; BPF: band-pass filter, SPF: short-pass filter; P: polarizer; PMT:

photomultiplier tube; PC: personal computer. (c) Pulse-lasing spectrum of the gain-switched InGaN VCSEL at room temperature. The inset shows the enlarged lasing spectra with various pump powers above the threshold, where the arrow indicates the increase in pump power. Note the spectral broadening toward short wavelengths as the pump power increases. (d) Plot of input vs. output of the InGaN VCSEL. (e) Enlarged spectrum of the InGaN VCSEL with pump power below the threshold. Solid curves are Gaussian fits of the modes. FWHM: full width at half maximum. FSR: free space range.

characterize blue-wavelength pulses produced by semiconductor lasers, the advantages of fine temporal resolution and high $\mathrm{S} / \mathrm{N}$ ratio are suited for our purpose.

In this study, we prepared a nitride-based VCSEL with 10 periods of InGaN/GaN quantum wells (QWs). For accurate evaluation of the pulse widths and shapes of the gain-switched output pulses, we used a time-resolved measurement system based on up-conversion method, which attained a resolution of $0.12 \mathrm{ps}$. We demonstrated that ultra-short pulses, as short as $2.2 \mathrm{ps}$ at $3.4 \mathrm{~K}$ and $4.0 \mathrm{ps}$ at room temperature in full width at half maximum (FWHM), were generated from the gain-switched InGaN VCSEL via impulsive optical pumping, without any post-processing. These record-breaking short blue pulses demonstrate the superiority of gain-switched nitridebased VCSELs as compact and reliable ultrafast blue light sources. In addition, our successful assessment demonstrates that the upconversion technique is indeed useful for characterizing ultra-short blue pulses generated by semiconductor lasers.

\section{Results}

Figure 1a shows a schematic of the structure of the InGaN VCSEL. It consists of 10-period InGaN/GaN quantum wells (QWs) and two $\mathrm{Ta}_{2} \mathrm{O}_{5} / \mathrm{SiO}_{2}$ distributed Bragg reflectors (DBRs). A description of the fabrication process can be found in our previous work ${ }^{25}$. Figure $1 \mathrm{~b}$ diagrams the experimental apparatus for the time-resolved up-conversion measurements. A detailed explanation of the system is given in the methods section below. The sample in a cryostat was optically pumped with a $1-\mathrm{kHz}, 400-\mathrm{nm}$, and $300-\mathrm{fs}$ pulse laser beam with a spot size of $\sim 50 \mu \mathrm{m}$. The output from the InGaN VCSEL was observed with a resolution of 0.12 ps. The lasing spectra of the gain-switched output pulses were characterized through a spectrometer system that employed a liquid-nitrogen-cooled chargecoupled device (CCD) with a spectral resolution of $0.15 \mathrm{~nm}$.

Figure 1c shows a time-integrated emission spectrum from the InGaN VCSEL, obtained with an optical pump power of $300 \mu \mathrm{W}$ at room temperature. Here, single-mode lasing at $425.7 \mathrm{~nm}$ is apparent. The single-mode lasing persisted even at elevated pumping power. The plot of the input vs. output powers shown in Fig. 1d demonstrates that the pulse-lasing threshold was $150 \mu \mathrm{W}$. The average output power with an pump power of $300 \mu \mathrm{W}$ was measured to be in an order of nW. Figure 1e shows an enlarged emission spectrum from the InGaN VCSEL operated with an optical pump power below the threshold. This plot shows that the free spectral range (FSR, i.e., the spacing between two adjacent cavity modes) is around $10.8 \mathrm{~nm}$, from which we estimate the cavity length $L$ to be $2.6 \mu \mathrm{m}$. The spectral width of the optical mode centered at $425.7 \mathrm{~nm}$ is $1.0 \mathrm{~nm}$; we estimate that its cavity quality factor is 425.7 , and that the photon lifetime within the cavity is approximately $0.1 \mathrm{ps}$ (see Supplementary Information).

Figure 2a shows the waveform of the output pulses from the gainswitched InGaN VCSEL at room temperature, with an excitation power of $300 \mu \mathrm{W}$, which is about two times greater than the lasing threshold. The InGaN VCSEL was optically pumped at $0 \mathrm{ps}$, and the gain-switched pulses were generated with a delay time. The shortest pulse width of these gain-switched pulses was found to be $4.0 \mathrm{ps}$, 

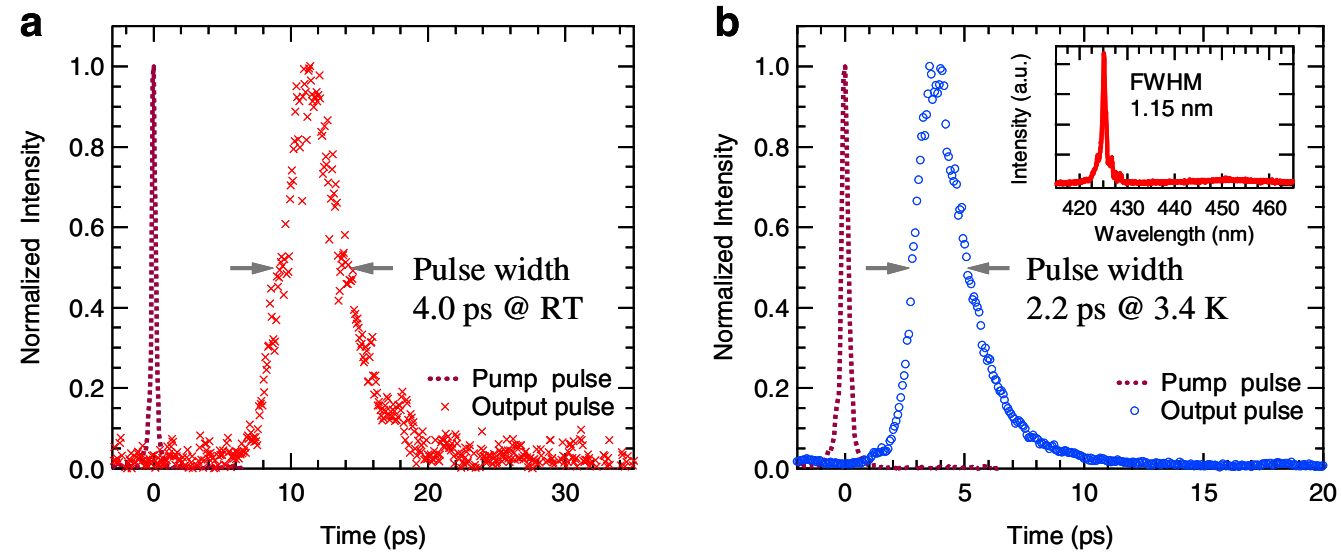

Figure $2 \mid$ Waveforms of the gain-switched pulses from the InGaN VCSEL at (a) room temperature and (b) 3.4 K measured via up-conversion method. The inset shows the lasing spectrum at $3.4 \mathrm{~K}$. The cross-correlation waveforms of the pump pulses, which was observed without the band-pass filter shown in Fig. 1b, are also plotted. The optical time delay arising in the filter is compensated for in these waveforms.

with a delay time of 11.4 ps. The measured waveform of the 300 -fs pump pulses indicates the fine temporal resolution of the up-conversion measurement system.

Figure $2 \mathrm{~b}$ shows the time-resolved waveform of the gain-switched pulses generated from the InGaN VCSEL at $3.4 \mathrm{~K}$, with an excitation power about 3 times higher than the lasing threshold of $80 \mu \mathrm{W}$. The pulse width was found to be 2.2 ps with a delay time of 3.9 ps. So far, this is the shortest pulse width that has been observed in gainswitched semiconductor blue lasers.

\section{Discussion}

In Fig. 3, the waveforms of these gain-switched pulses are plotted again in a logarithmic scale. Clear exponential rises and decays of these pulses, which are the typical characteristics for gain-switched pulses ${ }^{17,26}$, are apparent. The rise times, the delay times, the decay times, and the pulse widths of these gain-switched short pulses were obtained from a fitting analysis, and these are summarized in the top panel of Fig. 3. In this way, the characteristic profiles of the gainswitched pulses were accurately evaluated. Such a successful detailed analysis is made possible by the high $\mathrm{S} / \mathrm{N}$ ratio intrinsic to the upconversion technique.

\begin{tabular}{|l|l|c|c|c|}
\hline Temp. & $\begin{array}{l}\text { Pulse } \\
\text { width }\end{array}$ & $\begin{array}{c}\text { Rise } \\
\text { time }\end{array}$ & $\begin{array}{c}\text { Decay } \\
\text { time }\end{array}$ & $\begin{array}{c}\text { Delay } \\
\text { time }\end{array}$ \\
\hline $3.4 \mathrm{~K}$ & $2.2 \mathrm{ps}$ & $0.8 \mathrm{ps}$ & $1.9 \mathrm{ps}$ & $3.9 \mathrm{ps}$ \\
\hline $300 \mathrm{~K}$ & $4.0 \mathrm{ps}$ & $1.6 \mathrm{ps}$ & $2.9 \mathrm{ps}$ & $11.4 \mathrm{ps}$ \\
\hline
\end{tabular}

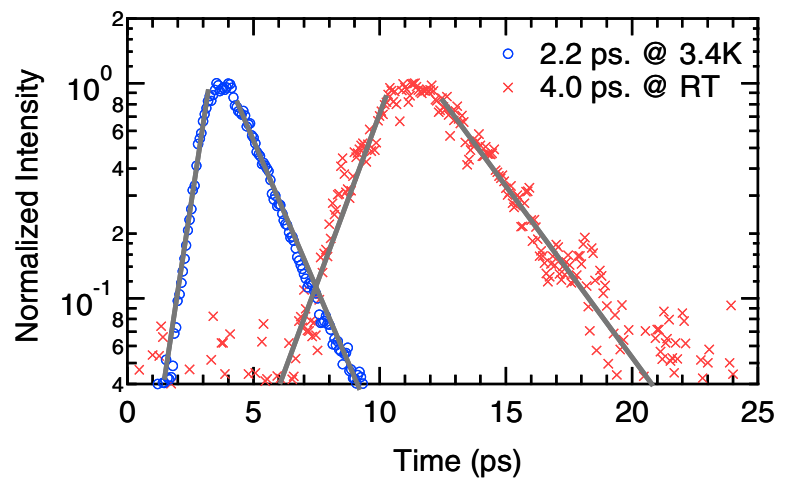

Figure 3 Waveforms of the gain-switched pulses from the InGaN VCSEL plotted in a logarithmic time scale. Note the clear exponential rise and decay portions of the pulses. The top panel summarizes the rise times and decay times as well as the pulse widths and delay times of these pulses.
The 4.0-ps pulses obtained at room temperature from the 10-QW VCSEL are very short, as compared with previously reported 6.0-ps gain-switched pulses generated from a 3-QW VCSEL that was prepared similarly ${ }^{27}$. According to our previous works ${ }^{17,26}$, the pulse width of gain-switched pulses is in general limited by the saturation modal gain of the lasers. Thus, we conclude that the increased number of quantum wells enhanced the modal gain, which shortened the gain-switched pulses. Note also that shorter 2.2-ps pulses were obtained at a lower temperature. We attribute this pulse shortening to the increase of gain at low temperatures which is typical in semiconductor lasers. These findings indicate that further enhancement of modal gain by simply increasing the number of quantum wells should be effective for the direct generation of even shorter pulses.

The decay times measured from the 10-QW VCSEL were much longer than the 0.1-ps photon lifetime. Thus, the decay times were not limited by the photon lifetime. Previous studies on a 3-QW VCSEL $^{27,28}$ showed that the gain-switched pulses were strongly chirped and had a slow-decay component on the long wavelength side, which actually limits decay time. In the lasing spectra of the 10QW VCSEL shown in the inset of Fig. 1c, a spectral broadening towards short wavelengths is apparent. This spectral tendency is very similar to the characteristics reported for the 3-QW VCSEL ${ }^{27,28}$. Therefore, we suspect that the gain-switched pulses from the 10QW VCSEL should also be strongly chirped and include a slow component. Although the origin of this slow component has not yet been made clear, it is necessary to reduce this component to shorten the decay time.

As it is shown in the inset of Fig. 2b, the spectral width and the center wavelength of the 2.2-ps pulses at the lower temperature were $1.15 \mathrm{~nm}$ and $425 \mathrm{~nm}$, respectively; the large time-bandwidth product of 4.2 indicates that these pulses are not Fourier-transform-limited. In such a case, some post-processing can still be useful to shorten the pulse width, though direct pulse generation is favorable for the sake of simplicity. For example, the slow component at longer wavelengths can be cut off by spectral filtering. Chirp-compression techniques should also be effective for shortening such strongly chirped pulses.

In summary, we fabricated a nitride-based VCSEL consisting of 10 -period InGaN/GaN quantum wells in order to study short blue pulse generation via gain-switching. In order to accurately measure the pulse widths and the shapes of the gain-switched output pulses from the InGaN VCSEL, we constructed an up-conversion measurement system with time resolution of $0.12 \mathrm{ps}$. We demonstrated the generation of blue pulses, as short as 2.2 ps at $3.4 \mathrm{~K}$ and 4.0 ps at room temperature, from the gain-switched InGaN VCSEL through optical pumping, without any post-processing. These record-break- 
ing short blue pulses confirm the superiority of gain-switched nitride-based VCSELs as compact and reliable ultra-short blue light sources. In addition, this successful assessment demonstrates that the up-conversion technique is indeed useful for characterizing ultrashort blue pulses from semiconductor lasers.

\section{Methods}

A schematic of the up-conversion experimental apparatus is shown in Fig. 1b. The 120 -fs $800-n m$ pulsed light source was emitted from a regenerative amplifier (Spectra Physics, Spitfire) seeded by a mode-locked Ti:sapphire laser (Spectra Physics, TSUNAMI 3160C). The VCSEL was mounted in a cryostat, and was optically excited by femtosecond pulses at $400 \mathrm{~nm}$, which were second-harmonic generations (SHG) of the 800 -nm fundamental wave, generated with a type-I phase-matching $\beta-\mathrm{BaB}_{2} \mathrm{O}_{4}$ $(\mathrm{BBO}) \operatorname{crystal}\left(\theta=29.3^{\circ}, \phi=0^{\circ}\right.$, thickness $\left.=0.5 \mathrm{~mm}\right)$. The duration of these pump pulses was measured to be about $300 \mathrm{fs}$, which is slightly broadened due to frequency chirping. The pump pulses were focused with a quartz lens having focal length of $60 \mathrm{~mm}$, and the excitation spot size was measured to be $\sim 50 \mu \mathrm{m}$ in diameter. Diffused pump light from the sample surface was completely removed by a band-pass filter $\left(\lambda_{\text {pass }}=447 \pm 30 \mathrm{~nm}\right.$, O.D. $\left.>6\right)$. The gain-switched pulses around $425 \mathrm{~nm}$, generated from the InGaN VCSEL, were overlapped with the $800-\mathrm{nm}$ gate pulses on a second type-I phase-matching BBO crystal $\left(\theta=44.3^{\circ}, \phi=0^{\circ}\right.$, thickness $\left.=0.1 \mathrm{~mm}\right)$ to generate an ultraviolet sum-frequency around $275 \mathrm{~nm}$. A short-pass filter $\left(\lambda_{\text {pass }}<\right.$ $340 \mathrm{~nm}$, O.D. $>6$ ) and a Glan-laser calcite polarizer were used to remove stray light. The wavelength-integrated intensity of the up-converted light (sum-frequency) was detected with a photomultiplier tube (Hamamatsu Photonics, R212) and recorded by a lock-in amplifier system. The measured temporal profile of the up-converted light corresponds to the waveform of the gain-switched pulses from the InGaN VCSEL. The time resolution of this measurement system was determined by the 0.12 -ps pulse duration of the gate pulses. The time-integrated lasing spectra of the gain-switched output pulses were characterized using a spectrometer system that employed a liquid nitrogen-cooled charge-coupled device (CCD) with spectral resolution of $0.15 \mathrm{~nm}$.

1. Ponce, F. A. \& Bour, D. P. Nitride-based semiconductors for blue and green lightemitting devices. Nature 386, 351-359 (1997).

2. Rigby, P. The future is looking blue. Nature 384, 610 (1996)

3. Gee, S. \& Bowers, J. E. Ultraviolet picosecond optical pulse generation from a mode-locked InGaN laser diode. Appl. Phys. Lett. 79, 1951 (2001).

4. Vasil'ev, P. P. et al. Mode locking in monolithic two-section InGaN blue-violet semiconductor lasers. Appl. Phys. Lett. 102, 121115 (2013).

5. Kuramoto, M. et al. Enormously high-peak-power optical pulse generation from a single-transverse-mode GaInN blue-violet laser diode. Appl. Phys. Lett. 96 , 051102 (2010).

6. Parthenopoulos, D. A. \& Rentzepis, P. M. Three-dimensional optical storage memory. Science 245, 843-845 (1989).

7. Anscombe, N. Data storage: Blue laser battle. Nat. Photon. 2, 393 (2008).

8. Tashiro, S. et al. Volumetric optical recording using a $400 \mathrm{~nm}$ all-semiconductor picosecond laser. Appl. Phys. Express 3, 102501 (2010)

9. Kawakami, R. et al. Visualizing hippocampal neurons with in vivo two-photon microscopy using a $1030 \mathrm{~nm}$ picosecond pulse laser. Sci. Rep. 3, 1014 (2013).

10. Yokoyama, H. et al. Two-photon bioimaging with picosecond optical pulses from a semiconductor laser. Opt. Express 14, 3467-3471 (2006).

11. Kono, S. et al. $12 \mathrm{~W}$ peak-power 10 ps duration optical pulse generation by gain switching of a single-transverse-mode GaInN blue laser diode. Appl. Phys. Lett. 93, 131113 (2008)

12. Oki, T. et al. Generation of over $10-\mathrm{W}$ peak-power picosecond pulses by a gainswitched AlGaInN-based self-pulsating laser diode. Appl. Phys. Express, 2, 032101 (2009).

13. Chen, S. Q. et al. Blue 6-ps short-pulse generation in gain-switched InGaN vertical-cavity surface-emitting lasers via impulsive optical pumping. Appl. Phys. Lett. 101, 191108 (2012).

14. Chen, S. Q. et al. Dynamics of short-pulse generation via spectral filtering from intensely excited gain-switched $1.55-\mu \mathrm{m}$ distributed-feedback laser diodes. Opt. Express 21, 10597-10605 (2013).
15. Wada, K. et al. Pulse-shaping of gain-switched pulse from multimode laser diode using fiber Sagnac interferometer. Opt. Express. 16, 19872-19881 (2008).

16. Takada, A., Sugie, T. \& Saruwatari, M. High-speed picosecond optical pulse compression from gain-switched 1.3- $\mu$ m distributed feedback-laser diode (DFBLD) through highly dispersive single-mode fiber. J. Lightwave Technol. 5, 1525-1533 (1987).

17. Chen, S. Q. et al. Analysis of gain-switching characteristics including strong gain saturation effects in low-dimensional semiconductor lasers. Jpn. J. Appl. Phys. 51, 098001 (2012).

18. Iga, K. Surface-emitting laser-Its birth and generation of new optoelectronics field. IEEE J. Select. Topics Quantum Electron. 6, 1201-1215 (2000).

19. Krestnikov, I. L. et al. Room-temperature photopumped InGaN/GaN/AlGaN vertical-cavity surface-emitting laser. Appl. Phys. Lett. 75, 1192-1194 (1999).

20. Someya, T. et al. Room temperature lasing at blue wavelengths in gallium nitride microcavities. Science 285, 1905-1906 (1999).

21. Suemoto, T. et al. Access to hole dynamics in graphite by femtosecond luminescence and photoemission spectroscopy. Phys. Rev. B. 87, 224302 (2013).

22. Matsuoka, T. et al. Real-time capturing of the nuclear wave-packet shape in selftrapped excitons. Phys. Rev. Lett. 91, 247402 (2003).

23. Shah, J. Ultrafast luminescence spectroscopy using sum frequency generation. J. Quantum Electron. 24, 276-288 (1988).

24. Hasnain, G. et al. Electrically gain-switched vertical-cavity surface-emitting lasers. IEEE. Photo. Tech. Lett. 4, 6-9 (1992).

25. Liu, W.-J. et al. Low threshold lasing of GaN-based VCSELs with sub-nanometer roughness polishing. IEEE Photo. Tech. Lett. 25, 2014-2017 (2013)

26. Ito, T. et al. Transient hot-carrier optical gain in a gain-switched semiconductor laser. Appl. Phys. Lett. 103, 082117 (2013).

27. Chen, S. Q. et al. Gain-switching dynamics in optically pumped single-mode InGaN vertical-cavity surface-emitting lasers. Opt. Express 22, 4196-4201 (2014).

28. Chen, S. Q. et al. Spectral dynamics in picosecond gain-switched pulses from nitride-based vertical-cavity surface-emitting lasers. Sci. Rep. 4, 4325 (2014).

\section{Acknowledgments}

This work was partially supported by KAKENHI No. 20104004 from MEXT, KAKENHI No. 23360135 and No. 26390075 from JSPS, the Photon Frontier Network Program of MEXT, JST-SENTAN, and JST-CREST in Japan. S.C. is thankful for the supports of the Recruitment Program of Global Experts (1000 Talent Plan) of China, and the Program for Professor of Special Appointment (Eastern Scholar) at Shanghai Institutions of Higher Learning.

\section{Author contributions}

H.A., M.Y. and S.C. conceived the project. W.L. and B.Z. fabricated the samples. A.A. and T.S. constructed the experimental apparatus. S.C., A.A. and T.I. contributed equally to this paper by characterizing the samples, analyzing the data and writing the manuscript under the supervision of H.A., M.Y. and T.S. All authors participated in discussion and commented on the manuscript.

\section{Additional information}

Supplementary information accompanies this paper at http://www.nature.com/ scientificreports

Competing financial interests: The authors declare no competing financial interests.

How to cite this article: Asahara, A. et al. Direct generation of 2-ps blue pulses from gain-switched InGaN VCSEL assessed by up-conversion technique. Sci. Rep. 4, 6401; DOI:10.1038/srep06401 (2014)

This work is licensed under a Creative Commons Attribution-NonCommercialShareAlike 4.0 International License. The images or other third party material in this article are included in the article's Creative Commons license, unless indicated otherwise in the credit line; if the material is not included under the Creative Commons license, users will need to obtain permission from the license holder in order to reproduce the material. To view a copy of this license, visit http:// creativecommons.org/licenses/by-nc-sa/4.0/ 